\title{
Temperature-Dependent Second Shell Interference in the First Shell Analysis of Crystalline InP X-ray Absorption Spectroscopy Data
}

\author{
Claudia S. Schnohr ${ }^{1}$, Leandro L. Araujo ${ }^{2}$, and Mark C. Ridgway ${ }^{3}$ \\ ${ }^{1}$ Institut für Festkörperphysik, Friedrich-Schiller-Universität Jena, Max-Wien-Platz 1, 07743 Jena, Germany \\ ${ }^{2}$ Instituto de Física, Universidade Federal do Rio Grande do Sul, Porto Alegre, 91501-970, Brazil \\ ${ }^{3}$ Research School of Physics and Engineering, The Australian National University, Canberra, ACT 0200, Australia
}

(Received May 20, 2014; accepted July 5, 2014; published online August 7, 2014)

\begin{abstract}
Analysing only the first nearest neighbour (NN) scattering signal is a commonly used and often successful way to treat extended X-ray absorption fine structure data. However, using temperature-dependent measurements of InP as an example, we demonstrate how this approach can lead to erroneous first NN structural parameters in systems with a weak first but strong second $\mathrm{NN}$ scatterer. In such cases, particularly low temperature data may suffer from an overlap of first and second NN scattering signals caused by the Fourier transformation (FT) even if the dominant peaks appear to be well separated. The first NN structural parameters then vary as a function of the FT settings if only the first NN scattering contribution is considered in the analysis. Although this variation is small, it can also lead to significant differences in other calculated properties such as the Einstein temperature. We demonstrate that these variations can be avoided either by choosing an appropriate FT window or by including the scattering contributions of higher shells in the analysis. The latter is achieved by a path fitting approach and yields structural parameters independent of the FT settings used.
\end{abstract}

\section{Introduction}

Extended X-ray absorption fine structure (EXAFS) spectroscopy is a powerful and versatile technique for structural analysis and is widely used in physics, chemistry, material science, geology, environmental science, and biology. ${ }^{1-4)}$ The first step in EXAFS data analysis is usually to isolate the oscillating fine structure from the absorption background and to convert the energy scale into the photoelectron wavenumber $k$ scale. The fine structure $\chi(k)$ thus obtained is then Fourier transformed into radial space where different scattering contributions produce different peaks when plotting the magnitude of the Fourier transformed $\chi(R)$. A backtransformation can then be used to isolate single scattering contributions provided their signals are well separated in $R$-space.

This methodology has been extensively used to analyse the first nearest neighbour (NN) scattering contribution with the Ratio Method. ${ }^{5,6)}$ It yields the differences in structural parameters between the sample of interest and a known reference. The Ratio Method is thus a model independent approach and does not need to assume any a priori structure. However, its major limitation is the need to have scattering contributions well isolated in radial space which usually limits the analysis to the first coordination shell. The first shell itself must consist of one element only with all atoms situated at similar distances from the absorber such that the first NN contribution can be described by a single scattering path. Crystalline group-IV, III-V, and II-VI semiconductors, for instance, fully meet this requirement. Temperature-dependent studies of these materials are thus ideal candidates for an analysis using the Ratio Method. ${ }^{7)}$ However, even in such cases care has to be taken with regard to the required isolation of the first $\mathrm{NN}$ scattering contribution in $R$-space as we will demonstrate for the specific example of InP.

An alternative and also widely used method of analysis is provided by the path fitting approach as realized, for example, by the IFEFFIT $\operatorname{code}^{8)}$ and the corresponding user interfaces AtHENA and ARTEMIS. ${ }^{9)}$ It is based on the cumulant expansion of the different single and multiple scattering paths and thus requires the use of a model structure. Its major advantage, however, is the ability to analyse the structural parameters beyond the first coordination shell where several single and multiple scattering paths typically overlap in $R$ space. Path fitting is also capable of analysing materials with a mixed first NN shell such as amorphous InP where the In atoms have both $\mathrm{P}$ and In first NN. ${ }^{10)}$

Despite the ability to include higher shells in the fitting procedure, the analysis is often reduced to the first NN shell even for a path fitting methodology. The reason for this is the widely held believe that scattering contributions originating from the first and higher coordination shells are well isolated in $R$-space. While this is certainly true for many systems, the assumption should be carefully tested by researchers for their specific materials of interest and for the parameters used in processing the data. Indeed, using temperature-dependent measurements of crystalline InP, ${ }^{11)}$ we will demonstrate how the second NN signal can interfere with the first shell analysis even for a seemingly well isolated first NN signal. Both the measurement temperature and the Fourier transformation (FT) parameters influence the effect of the second $\mathrm{NN}$ scattering contribution on the fitting parameters. Although the resulting differences in the first $\mathrm{NN}$ structural parameters are small, they may lead to erroneous values of other calculated properties such as the Einstein temperature. The validity of a pure first shell analysis, be it with the Ratio Method or a path fitting approach, is therefore strongly questionable in this case and, if at all, has to be carried out with extreme care. Similar effects will occur for other materials with a weak first but strong second NN scatterer.

\section{Data Acquisition and Analysis}

A single-crystal InP wafer was finely crushed and mixed with $\mathrm{BN}$ to give a powder sample suitable for EXAFS transmission measurements. Spectra of the In $K$ edge $(27.940 \mathrm{keV})$ were recorded at beamline NW10A of the Photon Factory, Japan, at eight different temperatures ranging from 20 to $295 \mathrm{~K}^{11}{ }^{11)}$ The raw data were processed in ATHENA to yield the fine structure contribution $\chi(k)$ as plotted in 

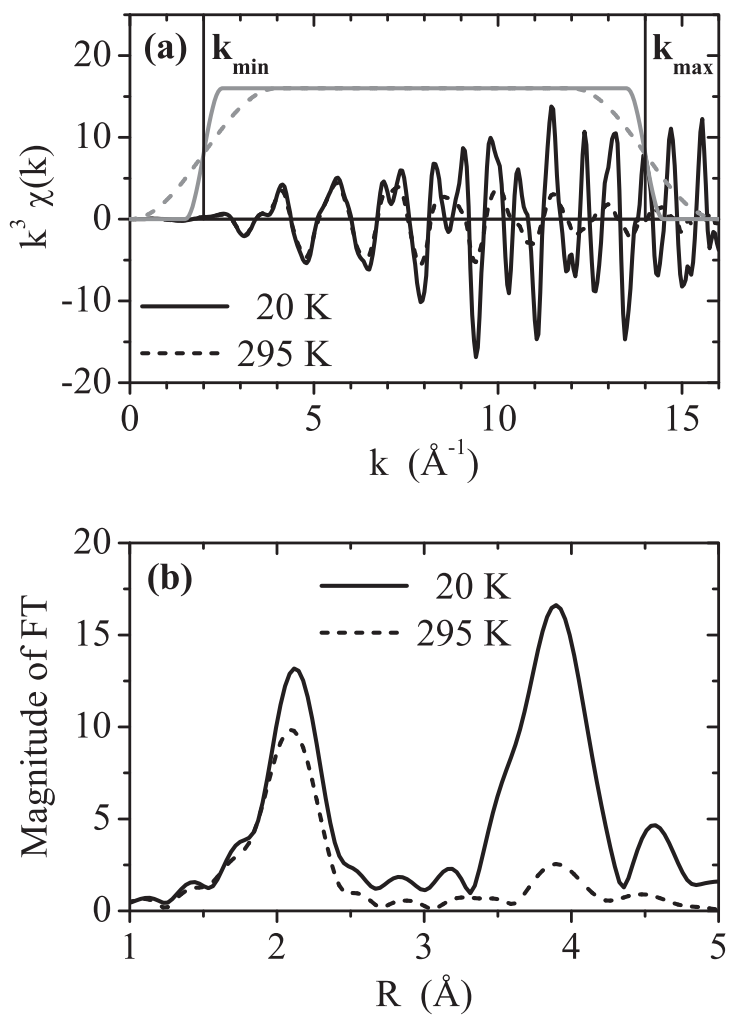

Fig. 1. (a) $k^{3}$-weighted EXAFS spectra of crystalline InP (thick black lines) measured at the In $K$ edge at $20 \mathrm{~K}$ (solid) and $295 \mathrm{~K}$ (dashed) versus the photoelectron wave number $k .^{11)}$ Two different Hanning window functions with $k_{\min }=2 \AA^{-1}$ and $k_{\max }=14 \AA^{-1}$ are also given (thick gray lines) for $d k=1 \AA^{-1}$ (solid) and $d k=4 \AA^{-1}$ (dashed). (b) Fourier transforms of the EXAFS data in panel (a) as a function of the non-phase-corrected radial distance $R$ using the window function with $d k=1 \AA^{-1}$.

Fig. 1(a) for the spectra taken at 20 and $295 \mathrm{~K}$ and multiplied by a $k$-weight of 3 . A FT was then performed using a Hanning window characterised by the minimum and maximum $k$ values, $k_{\min }$ and $k_{\max }$, respectively, and the width $d k$ of the window sides. Two specific window functions with $k_{\min }=2 \AA^{-1}, k_{\max }=14 \AA^{-1}$, and $d k=1$ or $4 \AA^{-1}$ are also shown in Fig. 1(a) while the magnitude of the resulting Fourier transforms using $d k=1 \AA^{-1}$ is plotted in Fig. 1(b). The peak at $R \sim 2.1 \AA$ stems from scattering at first $\mathrm{NN} P$ atoms. It appears to be well isolated from the features in the range $R \sim 3-5 \AA$ which result from the superposition of scattering at second NN In and third NN P atoms as well as some multiple scattering contributions. One could thus easily believe that a first shell analysis was straightforward in this material system.

To investigate whether the first NN scattering contribution can indeed be analysed independently of the higher shells, three different approaches were pursued. (i) The first shell signal of the Fourier transform obtained with a single $k$-weight of either 1, 2, or 3 was isolated by backtransformation using a Hanning window with $R_{\min }=1.5 \AA$, $R_{\max }=2.7 \AA$, and $d R=0.3 \AA$ and then analysed using the Ratio Method. (ii) Path fitting of the first shell signal was performed over the same $R$-range using ARTEMIs but applying multiple $k$-weights of 2 or 3 in the FT to reduce correlations between the fitting parameters. (iii) The first, second and third NN shells including a multiple scattering contribution were fitted in ARTEMIS over the range $R=1.5-4.9 \AA$ applying again $k$-weights of 2 or 3 in the FT. Details of the fitting procedure can be found in Ref. 11 .

In all three cases, $k_{\max }$ and $d k$ of the FT Hanning window were varied between 12.0 and $16.3 \AA$ and 0.5 and $4.0 \AA$, respectively. Note that the maximum possible value for $k_{\max }$ decreases with increasing $d k$ due to the finite data range. For each FT setting, the structural parameters of the first NN shell were evaluated, namely the interatomic distance, the EXAFS Debye-Waller factor and the asymmetry parameter. These properties represent the average value (first cumulant), the variance (second cumulant) and the asymmetry (third cumulant) of the one-dimensional interatomic distance distribution sampled by EXAFS. ${ }^{12)}$ The fourth cumulant was found to be negligible with both the Ratio Method and the path fitting approach and was thus not included in the analysis. In this paper, we will focus on the discussion of the EXAFS Debye-Waller factor and the related Einstein temperature.

\section{Results and Discussion}

\subsection{EXAFS Debye-Waller factor}

Figure 2 plots the EXAFS Debye-Waller factor $\sigma^{2}$ obtained from fitting the 20 and $295 \mathrm{~K}$ data for different values of $k_{\max }$. No variation of the values is observed if all shells are fitted and a sharp FT window with $d k=1 \AA^{-1}$ is used [Fig. 2(a)]. Applying a smooth FT window with $d k=4 \AA^{-1}$ yields the same results (not shown). This clearly demonstrates that the structural parameters determined from the path fitting procedure are independent of the FT settings if the higher shells are included in the fit.

The situation is, however, different if only the first NN shell is fitted. While no variation is observed for the high temperature values, the low temperature $\sigma^{2}$ show a pronounced oscillation with changing $k_{\max }$ if a sharp FT window with $d k=1 \AA^{-1}$ is used [Fig. 2(b)]. The amplitude of this oscillation decreases with increasing $k$-range but is still present at values as high as $k_{\max } \sim 16 \AA^{-1}$. Studying the spectra recorded at the intermediate temperatures, it becomes clear that the amplitude of the oscillation is strongest at $20 \mathrm{~K}$ and gradually decreases with increasing temperature.

In contrast, no variation of either the high or low temperature values is observed if a smooth FT window with $d k=4 \AA^{-1}$ is used [Fig. 2(c)]. Analysing this behaviour in more detail, we found that for $d k$ values of 0.5 and $1 \AA^{-1}$ oscillations of the low temperature $\sigma^{2}$ are present well up to $k_{\max } \sim 16 \AA^{-1}$ which represents the maximum possible $k$ range for this data set. For $d k=2 \AA^{-1}$ the oscillations are reduced but are still clearly visible especially for $k_{\max } \leq$ $14 \AA^{-1}$. Only for $d k=4 \AA^{-1}$ the oscillations vanish completely and the $\sigma^{2}$ values become independent of the FT $k$-range.

A similar behaviour is also observed using the Ratio Method. Applying a sharp FT window with $d k=1 \AA^{-1}$ results in $\Delta \sigma_{T}^{2}=\sigma_{T}^{2}-\sigma_{20 \mathrm{~K}}^{2}$ values, where $T$ denotes the measurement temperature, that strongly vary with both the maximum $k$-range used for FT and the maximum fitting range of the back-transformed data. In particular, using $k_{\max }=14$ or $12 \AA^{-1}$ yields $\Delta \sigma_{295 \mathrm{~K}}^{2}$ values that are systematically higher than those obtained with $k_{\max }=15$ or $13 \AA^{-1}$ in good agreement with Fig. 2(b). In contrast, applying a smooth FT window with $d k=4 \AA^{-1}$ yields values that are independent 


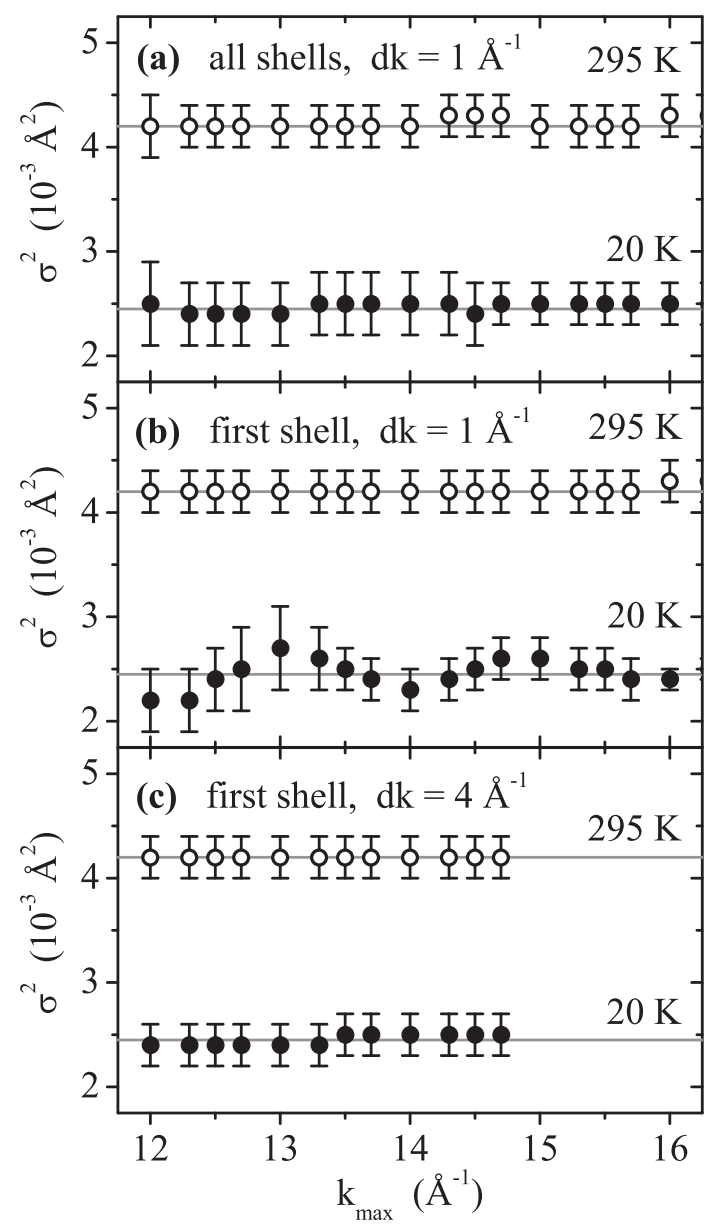

Fig. 2. EXAFS Debye-Waller-factor $\sigma^{2}$ of the first NN $\mathrm{P}$ path as a function of $k_{\max }$ used in the FT. The values are plotted for the lowest $(20 \mathrm{~K})$ and highest $(295 \mathrm{~K})$ measurement temperatures as obtained from (a) fitting the first, second, and third NN shell after using a sharp FT window with $d k=1 \AA^{-1}$, (b) fitting only the first NN shell after using a sharp FT window with $d k=1 \AA^{-1}$, and (c) fitting only the first NN shell after using a smooth FT window with $d k=4 \AA^{-1}$. All values were rounded to the first decimal digit.

of both $k$-range and fitting range within the experimental uncertainty. Furthermore, excellent agreement is observed between these values and those determined from a path fitting of all three first NN shells at all measurement temperatures.

\subsection{Second shell interference}

The oscillation of the EXAFS Debye-Waller factor and other structural parameters is only observed for a first shell analysis of the low temperature data. In contrast, no variation is found for either the high temperature data or when the first, second and third NN shell are included in the path fitting procedure. This suggests an influence of the second NN scattering contribution which is very strong at low temperatures but is heavily damped at room temperature due to thermal vibrations [Fig. 1(b)]. To test this hypothesis, the influence of the different FT settings on the various scattering contributions must be investigated. This can be achieved by the path fitting approach including the first, second and third NN shell in the fit and monitoring the fitted signal for each individual scattering path.

Figure 3 plots the magnitude of the Fourier transform versus radial distance $R$ for the experimental data, the

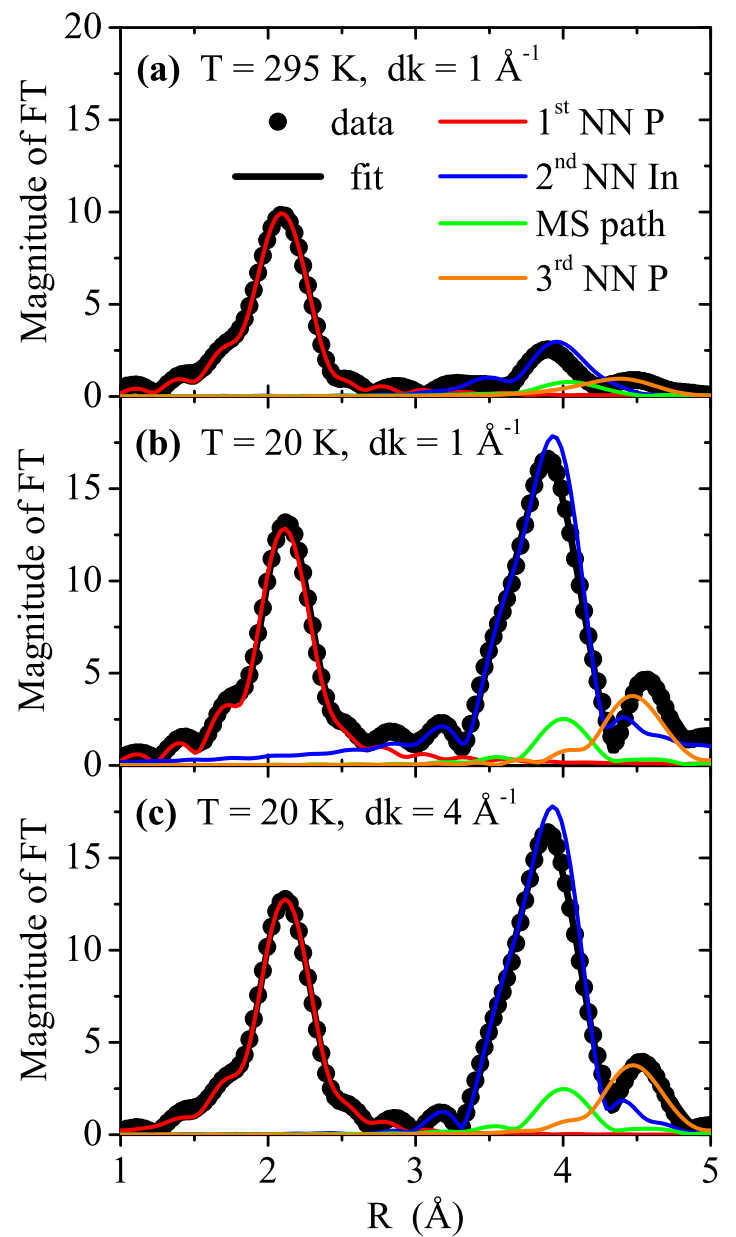

Fig. 3. (Color online) Magnitude of the Fourier transform versus radial distance $R$ for the experimental data (black symbols), the resulting fit including the first, second and third NN shell (black line) and the individual scattering paths associated with first NN P (red line), second NN In (blue line) and third NN P (orange line). The second NN In - first NN P multiple scattering (MS) contribution is also plotted (green line). Panel (a) shows the data recorded at $T=295 \mathrm{~K}$ after applying a FT with $k_{\max }=14 \AA^{-1}$ and $d k=1 \AA^{-1}$ while panels (b) and (c) plot the data recorded at $T=20 \mathrm{~K}$ after applying a FT with $k_{\max }=14 \AA^{-1}$ and $d k=1$ and $4 \AA^{-1}$, respectively.

resulting fit and the individual scattering paths using a maximum $k$-range of $k_{\max }=14 \AA^{-1}$. At room temperature, the scattering contributions of the higher shells are heavily damped and no overlap with the first NN signal is observed [Fig. 3(a)]. Fitting only the first shell thus yields the same first NN structural parameters as fitting the first, second and third shell together.

The situation is clearly different at $T=20 \mathrm{~K}$ if a sharp window with $d k=1 \AA^{-1}$ is used in the FT [Fig. 3(b)]. The second NN scattering contribution now exhibits a nonnegligible magnitude even at radial distances typically associated with only the first $\mathrm{NN}$ signal. The experimental data in this region $R<3 \AA$ is thus a superposition of first and second NN scattering. Including only the first shell, i.e., the first $\mathrm{NN}$ scattering path, in the fitting procedure therefore yields slightly different first $\mathrm{NN}$ structural parameters than fitting all three $\mathrm{NN}$ shells where the second NN scattering path is also included. Note that changing the maximum $k$-value used in the FT does affect the appearance and position of the small features at $R=$ 
2.5-3.5 $\AA$ between the two dominant peaks. In contrast, it does not change the magnitude of the second $\mathrm{NN}$ scattering signal in the region $R=1.5-2.5 \AA$ typically associated with the first $\mathrm{NN}$ shell.

These tails in the scattering signal after FT are well known and are often referred to as "ripple" or "noise". However, they are part of the physical signal and originate from the fact that the $k$-space data used for FT is limited in range. ${ }^{2,3)}$ Applying a step-like window function yields the strongest tails while very smooth window functions such as a Gaussian function yield only minor tails. Consequently, the magnitude of the second NN scattering signal for $R<3 \AA$ in Fig. 3(b) is strongly influenced by the width of the Hanning window sides. While sharp windows with $d k=0.5$ or $1 \AA^{-1}$ produce significant tails [Fig. 3(b)], the second NN signal for $R<3 \AA$ is reduced for $d k=2 \AA^{-1}$ and vanishes completely for a very smooth window with $d k=4 \AA^{-1}$ as shown in Fig. 3(c). In this case, fitting only the first NN shell results again in the same first NN structural parameters as fitting first, second, and third shell together. Interestingly, a Hanning window width of $d k=1$ or $2 \AA^{-1}$ is often considered sufficiently smooth to avoid unwanted signal tails ${ }^{2,4)}$ whereas our results clearly demonstrate that a significant signal overlap from first and second NN scattering paths is still present in this case for our low temperature InP data. This explains why the low temperature $\sigma^{2}$ values in Figs. 2(b) but not 2(c) differ from those of Fig. 2(a) while the high temperature values are in excellent agreement for all three cases.

A deviation of the first $\mathrm{NN}$ structural parameters deduced with a first shell fit from those obtained analysing all three shells thus only occurs for low temperature data and sharp FT windows where a tail of the second NN scattering contribution overlaps with the first NN signal. The oscillation of the $20 \mathrm{~K}^{2}$ values in Fig. 2(b) suggests that the detailed nature of this second shell contribution depends on the FT $k$-range. Figure 4 plots the real part of the Fourier transform versus radial distance $R$ in the range of the first NN peak. The $20 \mathrm{~K}$ experimental data, the resulting fit and the first and second NN scattering paths obtained using a sharp FT window with $d k=1 \AA^{-1}$ are plotted for $k_{\max }=13$ and $14 \AA^{-1}$ in panel (a) and (b), respectively. Obviously, the magnitude of the second NN signal is not affected by the choice of $k$-range as already mentioned above. In contrast, the phase relation between the first and second $\mathrm{NN}$ scattering contributions is clearly different in the two cases. Detailed analysis shows that the phase difference between first and second $\mathrm{NN}$ path varies by almost $180^{\circ}$ at $R \sim$ $2.1 \AA$. Depending on $k_{\max }$, the second NN signal is thus either in phase or out of phase with the first NN scattering contribution leading to an overall fit that is higher or lower than the first NN path. A fit that does not include the second $\mathrm{NN}$ path therefore over- or underestimates the first $\mathrm{NN}$ scattering contribution and results in the observed oscillation of the structural parameters with the $k_{\max }$ value used in the FT.

\subsection{Temperature dependence}

It could be argued that the variation of the low temperature $\sigma^{2}$ values observed in Fig. 2(b) is still within the uncertainty (error bars) reported from the fit and that it is thus of no further consequence. On the contrary, we will now

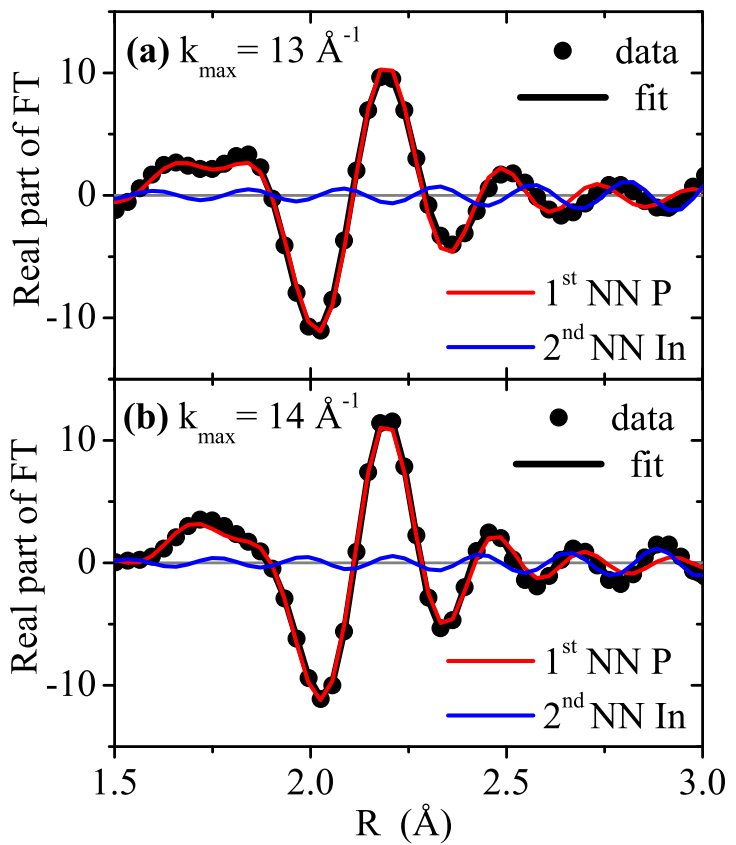

Fig. 4. (Color online) Real part of the Fourier transform versus radial distance $R$ for the experimental data recorded at $T=20 \mathrm{~K}$ (black symbols), the resulting fit including all three NN shells (black line) and the first NN P (red line) and second NN In (blue line) scattering paths. The maximum $k$-value used in the FT was $k_{\max }=13$ and $14 \AA^{-1}$ in panel (a) and (b), respectively, while a sharp FT window with $d k=1 \AA^{-1}$ was applied in both cases.

demonstrate that this oscillation of the structural parameters is indeed significant and may influence other calculated parameters such as the Einstein temperature.

The increase of the EXAFS Debye-Waller factor with increasing temperature is caused by thermal vibrations of the atoms which result in a broadening of the interatomic distance distribution. For compound semiconductors such as InP, the temperature dependence of $\sigma^{2}$ can be well described by a correlated Einstein model: ${ }^{11,13,14)}$

$$
\sigma^{2}=\frac{\hbar^{2}}{2 \mu k_{\mathrm{B}}} \frac{1}{\theta_{\mathrm{E}}} \operatorname{coth}\left(\frac{\theta_{\mathrm{E}}}{2 T}\right)+\sigma_{\text {static }}^{2} .
$$

Here $T$ is the temperature, $\hbar$ and $k_{\mathrm{B}}$ denote Planck's constant divided by $2 \pi$ and Boltzmann's constant, respectively, $\mu$ is the reduced mass of the absorber-backscatterer pair and $\theta_{\mathrm{E}}$ is the Einstein temperature. The latter represents a pivotal property characterising the vibrational behaviour of the material. Temperature-dependent EXAFS measurements thus provide one means of experimentally determining the Einstein temperature.

Figure 5 plots $\sigma^{2}$ versus temperature $T$ obtained from fitting only the first $\mathrm{NN}$ shell and applying a sharp FT window with $d k=1 \AA^{-1}$ and $k_{\max }=13$ or $14 \AA^{-1}$. As already discussed above, the differences between the $\sigma^{2}$ values resulting from the two different $k_{\max }$ settings are most pronounced at low temperatures and gradually vanish with increasing $T$. Despite the fact that the variation is similar to the uncertainty of the data, the resulting fits with the correlated Einstein model are significantly different (Fig. 5), corresponding to Einstein temperatures of $\theta_{\mathrm{E}}=408 \pm 7$ and $390 \pm 9 \mathrm{~K}$ for $k_{\max }=13$ or $14 \AA^{-1}$, respectively. Similarly, the static contribution amounts to $\sigma_{\text {static }}^{2}=(0.2 \pm 0.1) \times$ 


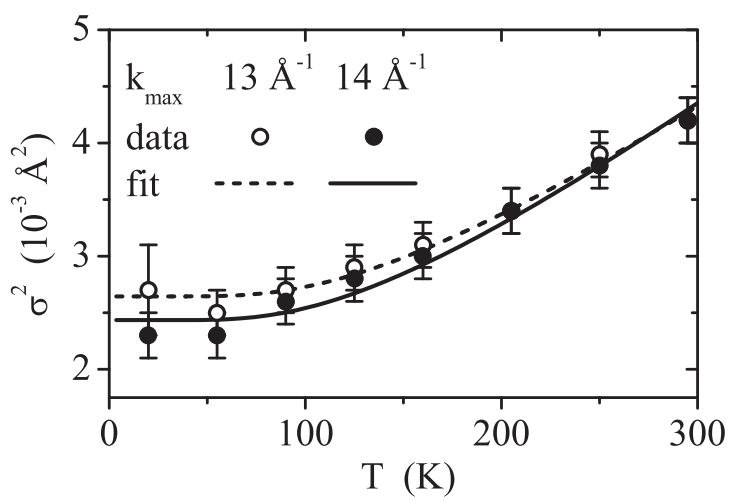

Fig. 5. EXAFS Debye-Waller-factor $\sigma^{2}$ of the first NN $\mathrm{P}$ path as a function of temperature $T$ obtained from a first shell fit of the data after applying a sharp FT window with $d k=1 \AA^{-1}$ and a maximum $k$-range of either $k_{\max }=13 \AA^{-1}$ (open symbols) or $k_{\max }=14 \AA^{-1}$ (full symbols). All values were rounded to the first decimal digit. The corresponding fits with the correlated Einstein model are plotted as dashed and solid line, respectively.

$10^{-3} \AA^{2}$ for the smaller $k_{\max }$ and to $\sigma_{\text {static }}^{2}=(-0.1 \pm 0.1) \times$ $10^{-3} \AA^{2}$ for the larger $k_{\max }$.

The dependence of $\theta_{\mathrm{E}}$ and $\sigma_{\text {static }}^{2}$ on the FT $k$-range in the case of a first shell fit together with a sharp FT window is further demonstrated in Fig. 6. The Einstein temperature varies between 382 and $409 \mathrm{~K}$ depending on $k_{\max }$ while the static contribution of the EXAFS Debye-Waller factor ranges between -0.3 and $0.2 \times 10^{-3} \AA^{2}$. The values $\theta_{\mathrm{E}}=395 \mathrm{~K}$ and $\sigma_{\text {static }}^{2}=0.0 \times 10^{-3} \AA^{2}$ obtained from fitting the first, second and third NN shell together are given as grey lines for comparison. For both $\theta_{\mathrm{E}}$ and $\sigma_{\text {static }}^{2}$, the variation is thus significantly larger than the uncertainty of the values determined from the fit of the $\sigma^{2}$ data. More importantly, the variation of nearly $30 \mathrm{~K}$ in $\theta_{\mathrm{E}}$ is larger than the difference of approximately $20 \mathrm{~K}$ between the Einstein temperatures of crystalline and amorphous InP. ${ }^{11)}$ While EXAFS is indeed capable of unravelling subtle structural differences, it is only possible to exploit this potential when systematic errors of calculated properties such as shown in Fig. 6 are successfully avoided.

A similar variation of $\theta_{\mathrm{E}}$ and $\sigma_{\text {static }}^{2}$ is also observed when fitting the $\Delta \sigma^{2}$ values obtained from the Ratio Method after applying a sharp FT window. In contrast, no variation is apparent for both the Ratio Method and the path fitting approach when a smooth FT window is used or when the higher NN shells are included in the path fitting procedure. In these cases, the results obtained with all three approaches are again in excellent agreement with each other.

Our findings clearly demonstrate how a small variation of the low temperature but not the high temperature EXAFS Debye-Waller factors can significantly alter the results calculated for other important properties such as the Einstein temperature. Careful investigation of the influence of all parameter settings on the final results is thus an absolute necessity for a reliable data analysis.

\section{Conclusions}

We have analysed temperature-dependent EXAFS measurements of crystalline InP at the In $K$ edge in three different ways. The first NN scattering contribution was evaluated with either the Ratio Method or a path fitting approach. Furthermore, the scattering contributions originating from the

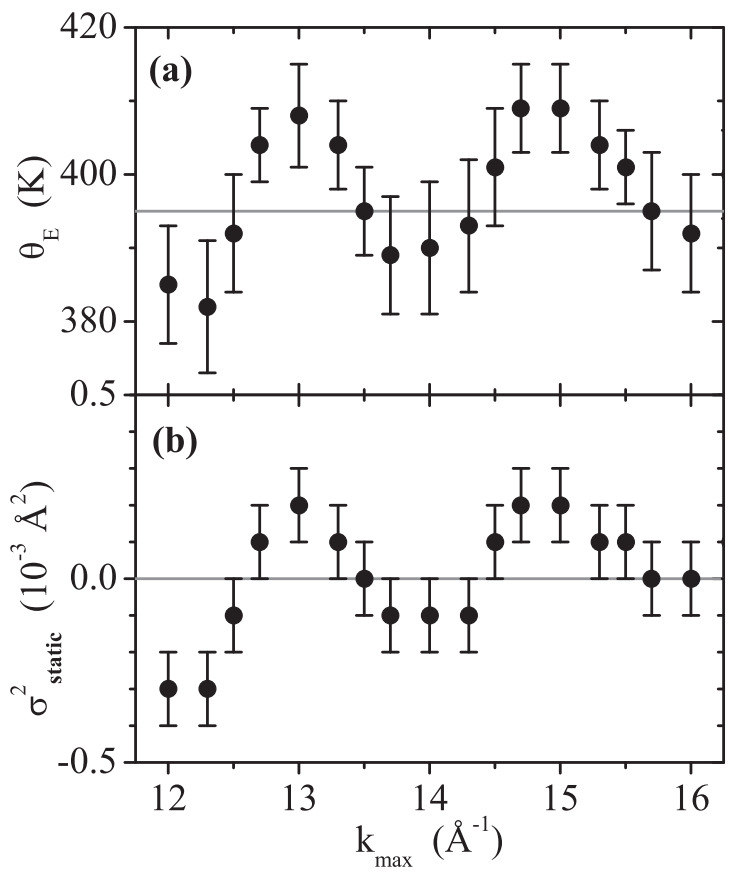

Fig. 6. (a) Einstein temperature $\theta_{\mathrm{E}}$ and (b) static contribution to the EXAFS Debye-Waller factor $\sigma_{\text {static }}^{2}$ as a function of $k_{\max }$ used in the FT. The values result from fitting the $\sigma^{2}$ data obtained from a first shell fit after applying a sharp FT window with $d k=1 \AA^{-1}$. The results obtained from fitting the first, second, and third shell together are given as grey lines for comparison. All $\sigma_{\text {static }}^{2}$ values were rounded to the first decimal digit.

first three NN shells were analysed simultaneously using again a path fitting approach. In all three cases, the first $\mathrm{NN}$ structural parameters, particularly the Debye-Waller factor $\sigma^{2}$, were evaluated as a function of the FT settings for different measurement temperatures.

A significant dependence of the low temperature $\sigma^{2}$ values on $k_{\max }$ of the FT is observed when only the first NN shell is analysed and a sharp FT window is used. In contrast, no variation is found when a very smooth FT window is applied. The high temperature structural parameters are independent of $k_{\max }$ for both sharp and smooth window functions. Similarly, neither the low nor high temperature results depend on the FT settings if the higher shells are included in the analysis. The uncertainties of structural parameters determined from these EXAFS measurements can thus be reduced (i) by choosing an appropriate FT window for a first shell analysis, although the requirements for an FT window to be appropriate may differ for each material studied, or (ii) by including scattering contributions from higher shells in a path fitting analysis which yields results independent of all FT settings.

The oscillation of the low temperature $\sigma^{2}$ values in the case of a first shell fit with a sharp FT window is caused by a tail of the second $\mathrm{NN}$ scattering contribution overlapping with the first NN signal. This second shell signal is not properly taken into account if only the first $\mathrm{NN}$ scattering path is included in the analysis leading to erroneous first $\mathrm{NN}$ structural parameters. In contrast, no overlap of first and second NN scattering is observed for very smooth FT windows or for spectra recorded near room temperature where the second NN scattering contribution is severely damped due to thermal vibrations. 
The first and second NN signals thus may or may not overlap depending on the measurement temperature and the FT settings. While the assumption of a well isolated first $\mathrm{NN}$ shell is certainly correct for many systems, it is clearly not true for EXAFS measurements of the In $K$ edge of crystalline InP where the first NN P is a rather weak scatterer while the second $\mathrm{NN}$ In is a comparatively strong scatterer. In this and other binary systems where the atomic numbers of the two components differ significantly, analysing only the first NN signal is thus problematic and extreme care must be taken to avoid influences of the FT settings. In contrast, structural parameters and calculated properties are independent of the FT details if scattering contributions from higher shells are properly considered during the analysis. The latter methodology thus presents a straightforward way of reducing the uncertainty of EXAFS results independent of the FT settings.

The variation of the low temperature $\sigma^{2}$ values for a first shell fit of our data using a sharp FT window is still within the uncertainty reported in the fit. Nevertheless, we have clearly demonstrated that even such a small variation can lead to significant differences in the vibrational parameters deduced from the temperature-dependent data. The Einstein temperature, in particular, varies by almost $30 \mathrm{~K}$ depending on the choice of $k_{\max }$ which is larger than the difference between the Einstein temperature of crystalline and amorphous InP. Seemingly harmless differences in the structural parameters induced by changing FT settings can thus have a strong impact on other properties and must be investigated rigorously in order to exploit the full potential of EXAFS as a powerful tool for structural analysis. We have demonstrated that these variations can indeed be avoided by choosing a very smooth FT window or by including higher shells in the path fitting analysis thus providing results independent of the FT settings.

\section{Acknowledgements}

We thank Paolo Fornasini for helpful and stimulating discussions. The authors were supported by the Carl-Zeiss Stiftung Germany, the Australian Research Council, and the Australian Synchrotron.

1) D. C. Koningsberger and R. Prins, X-ray Absorption: Principles, Applications, Techniques of EXAFS, SEXAFS and XANES (Wiley, New York, 1988).

2) S. D. Kelly, D. Hesterberg, and B. Ravel, Methods of Soil Analysis (Soil Science Society of America, Madison, WI, 2008) Part 5.

3) G. Bunker, Introduction to XAFS: A Practical Guide to X-ray Absorption Fine Structure Spectroscopy (Cambridge University Press, Cambridge, U.K., 2010)

4) S. Calvin, XAFS for Everyone (CRC Press, Boca Raton, FL, 2013)

5) G. Bunker, Nucl. Instrum. Methods Phys. Res. 207, 437 (1983).

6) G. Dalba, P. Fornasini, R. Grisenti, D. Pasqualini, D. Diop, and F. Monti, Phys. Rev. B 58, 4793 (1998).

7) N. Abd el All, B. Thiodjio Sendja, R. Grisenti, F. Rocca, D. Diop, O. Mathon, S. Pascarelli, and P. Fornasini, J. Synchrotron Radiat. 20, 603 (2013).

8) M. Newville, J. Synchrotron Radiat. 8, 322 (2001)

9) B. Ravel and M. Newville, J. Synchrotron Radiat. 12, 537 (2005).

10) C. S. Schnohr, P. Kluth, A. P. Byrne, G. J. Foran, and M. C. Ridgway, Phys. Rev. B 77, 073204 (2008).

11) C. S. Schnohr, P. Kluth, L. L. Araujo, D. J. Sprouster, A. P. Byrne, G. J. Foran, and M. C. Ridgway, Phys. Rev. B 79, 195203 (2009).

12) Note that while the path fitting approach immediately yields the absolute values of the cumulants of the real distance distribution, the Ratio Method provides the differences between the cumulants of the effective distance distribution of reference and sample. ${ }^{5)}$ The first cumulant of the real and effective distance distribution differs significantly whereas the differences for the second and third cumulants are usually negligible. ${ }^{6}$ To compare the results of both methodologies, the values obtained from the path fitting approach were recalculated to correspond to those provided by the Ratio Method.

13) T. Yokoyama, J. Synchrotron Radiat. 6, 323 (1999).

14) M. Vaccari and P. Fornasini, J. Synchrotron Radiat. 13, 321 (2006) 